\title{
Visual versus random-point percent cover estimations: 'objective' is not always better
}

\author{
Megan N. Dethier ${ }^{1, *}$, Elizabeth S. Graham ${ }^{2}$, Sarah Cohen ${ }^{3}$, Lucinda M. Tear ${ }^{4}$ \\ ${ }^{1}$ Friday Harbor Laboratories, University of Washington, Friday Harbor, Washington 98250, USA \\ ${ }^{2}$ Dept of Ecology and Evolutionary Biology, Princeton University, Princeton, New Jersey 08544, USA \\ ${ }^{3}$ Hopkins Marine Station, Stanford University, Pacific Grove, California 93950, USA \\ ${ }^{4}$ School of Fisheries, University of Washington, Seattle, Washington 98195, USA
}

\begin{abstract}
We compared the accuracy and repeatability of 2 common methods of estimating percentage covers of sessile organisms: visual estimates and random-point-quadrats (RPQ). Comparisons of estimates were made using both quadrats in the rocky intertidal zone and simulated quadrats drawn on a computer, where estimates could be compared with true, digitized percent cover values. In each case, visual estimates were found to be more repeatable (less within- and among-observer variation) and more accurate (closer to the true value as determined by digitizing) than the RPQ method. RPQs using 100 points were more accurate and less variable than those using 50 points, but were still less accurate (and much slower to carry out) than visual estimates. The RPQ method often missed rare species ( $<2 \%$ cover) altogether, but when it 'hit' them, values were usually overestimated. Visual estimates also tended to overestimate percent covers of species (although less than the RPQ method), especially uncommon ones. Thus although the probabilistic RPQ method is supposedly more objective and is statistically valid, visual estimates may give a more accurate representation of relative coverage of sessile organisms, and can reduce overall sampling error because they make increased sample sizes possible. Use of small subdivisions in quadrats, pre-field observer training, and a conscious effort to avoid bias are necessary to make the visual method valid and accurate.
\end{abstract}

\section{INTRODUCTION}

Terrestrial, aquatic, and marine ecologists often must quantify surface coverage of plants and sessile animals. Researchers have debated the relative advantages of visual (or 'ocular') estimates versus pointintercept methods for sampling in quadrats or other randomly selected plots (e.g. Greig-Smith 1964, Jones et al. 1980, Dethier 1984, Bonham 1989, Foster et al. 1991, Meese \& Tomich 1992). Objectivity, accuracy, precision (repeatability), efficiency, and sensitivity (e.g. to detection of rare species) are all important.

The broadly used random-point-quadrat (RPQ) method (described below) has 3 advantages: (1) it is objective, (2) its accuracy can be estimated theoretically based upon distributional assumptions about the populations being sampled, and (3) repeated samplings will converge on the true abundance value. The

- Address for correspondence: 620 University Road, Friday Harbor, Washington 98250 , USA disadvantages of the RPQ method include its limited measuring accuracy with one sample (dependent upon the number of random points used), its tendency to 'miss' rare species, and the considerable time involved in employing it. The visual estimation method has the disadvantage of being subjective, and since it is not a probabilistic sampling method, repeated samplings will not necessarily converge on the true value; however, its accuracy has seldom been tested. Foster et al. (1991) compared the RPQ method with a third method, involving photographing quadrats and then projecting the photographs onto a grid of points. They found that the photographic method 'missed' more taxa and underestimated organismal cover relative to the RPQ method, especially when organisms were multilayered. Meese \& Tomich (1992) photographed quadrats and then digitized the images, and found this to be more repeatable and less subject to observer bias than either a visual method or with point intercepts. However, without simultaneous (and time-consuming) mapping of organisms, in a photograph it is often diffi- 
cult to distinguish outlines of species of similar morphologies, making analysis of cover difficult with either digitizing or overlays of points.

The relative accuracies in estimating surface coverage (hereafter called 'percent cover') of these methods have not been compared for field data, since the 'true' percent covers of various species in a quadrat are difficult to assess. Our study was designed to compare visual estimates of percent cover with RPQ estimates, both from field data and from simulated plots. The latter comparison overcame the problem of not knowing the true percent cover, since we created on a computer 2-dimensional assemblages of 'species' whose exact percent covers could be measured by digitizing their areas. This study was not intended to address issues of sampling designs in general, but rather to compare the ability of 2 commonly used field techniques to accurately represent relative coverage of sessile organisms.

\section{METHODS}

Field. Field surveys to quantify cover of sessile organisms involve randomly selecting sites for quadrats, generating species lists, and estimating percent covers. We used both RPQ and visual methods to estimate the percent covers of sessile plants and animals in permanent $0.25 \mathrm{~m}^{2}(50 \times 50 \mathrm{~cm})$ quadrats on a waveexposed rocky shore in Washington, USA. Visual estimates were made with the aid of 25 small squares $(10 \times 10 \mathrm{~cm}$ each) marked off within the quadrat frame. Each small square 'filled' by a species was counted as $4 \%$ cover; often this technique required mentally 'grouping' organisms smaller than one full square and then counting the numbers of squares filled. This method eliminates the need for decision rules such as 'any square $>$ half filled is counted as filled'; instead, a square $3 / 4$ filled is simply $3 \%$ cover. Organisms filling $<1 / 4$ square $(<1 \%)$ were noted as 'rare', and given an arbitrary rating of $0.5 \%$ in the database. Since we were interested in all the layers of organisms, i.e. those on the rock as well as overlying algal canopies, we treated the system as 3-dimensional rather than looking only at the 2-dimensional canopy as in another methods comparison (Meese \& Tomich 1992); estimates of percent cover were made for each layer separately.

Random points (50 per quadrat) were projected onto a fine screen mesh held within a second quadrat frame. In the absence of any data on the possible regularity of distribution patterns, we considered it prudent to use random rather than systematic points. A metal rod was dropped through the appropriate coordinates on the mesh, and the organism contacted by the rod recorded; for multilayered quadrats, several species could be contacted by one point. Two observers (M.N.D. and S.C.) sampled each of 10 quadrats using each of the 2 methods over a period of $3 \mathrm{~d}$. Five were in the very high intertidal zone, and 5 in the mid zone. Since we had no way to measure which method was most accurate, i.e. which measured the 'true' coverage most effectively, we analyzed the data to see which method was most repeatable between the 2 observers; repeatability is considered to be crucial (Meese \& Tomich 1992). To do this, we investigated which method showed the smallest between-observer difference in percent cover values for each species recorded in each quadrat (hereafter called species-occurrences).

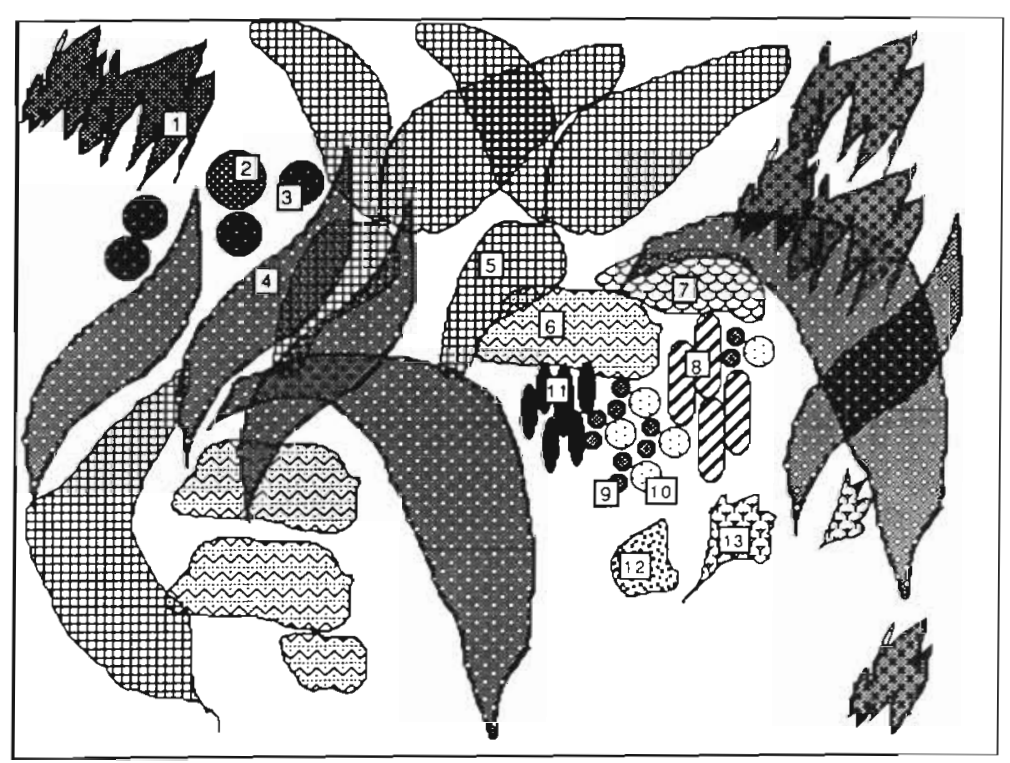

Fig. 1. A sample simulated 'low intertidal' quadrat, containing 13 'species' drawn to resemble real plants and animals (e.g. species $2=$ the anemone Anthopleura, $4=$ the red blade Dilsea, 6 = encrusting coralline algae, $8=$ goose neck barnacles, $11=$ the small mussel Mytilus) 
Simulation. Using Superpaint software on a Macintosh computer, we created 10 'intertidal quadrats' $(20 \times 25 \mathrm{~cm})$ containing different 'species', drawn to resemble real plants and animals in size (relative to the quadrat frame), shape, pattern of distribution (e.g. clumped like mussels, or scattered like algal blades), and percent cover. An example is shown in Fig. 1. Mathematically described shapes were not used, as they would have been too easy to assign a consistent percent cover using the visual method. Organisms were drawn to overlap each other, creating '3-dimensional' quadrats like those in the field. Five quadrats mimicked the high intertidal, and 5 the low intertidal, each with 10 to 13 species. For each of the 10 quadrats, we created 3 visually distinct versions ( $A, B$, and $C$ ) by haphazardly rearranging the 'organisms' in the quadrat, e.g. by moving a group of 'barnacles' and other species to new locations, but keeping all percent covers identical. This enabled us to record 3 different RPQ estimates for each quadrat (since the points contacted different organisms in each version), and 3 visual estimates for each quadrat (without the visual estimates being biased by the observer remembering previous estimates for a given set of organisms). Thus we obtained means and variances for the estimates produced by each of the methods. While 3 is a small number of replicates, it is not unrealistic for many field sampling programs, and the same degree of replication was used in comparing each of the methods.

RPQ estimates were produced as in the field, although here we used first 50 and then 100 random points. The points were marked on transparencies, with a different set of points used for each version of each quadrat.

Three different observers performed visual estimates using a $20 \times 25 \mathrm{~cm}$ quadrat frame marked off into $4 \times 5 \mathrm{~cm}$ rectangles. Observer 1 (M.N.D.) made estimates for all 3 (rearranged) versions of each quadrat ( 30 total); quadrats were shuffled by an independent party to minimize 'learning' from one version to another. Observers 2 and 3 estimated percent covers in Version A of each quadrat only (10 total). Observer 1 was the most experienced in the use of the visual estimation technique. For Observers 2 and 3, training consisted simply of a description of the method of using the small squares and of mentally 'grouping' scattered organisms.

The time required to sample a quadrat by each method was recorded after the observers had become familiar with the method. We determined the percentage of the total area covered by each species by digitizing using Image (version 1.22y) software.

\section{RESULTS}

\section{Field}

Out of 85 species-occurrences (both tidal heights pooled), the RPQ method 'missed' $19 \%$ (16) altogether. All these were less than $2 \%$ cover (according to the visual estimates), and none of the 50 random points happened to contact them. Of the remaining 69 occurrences, visual estimates of percent cover proved to be somewhat more repeatable (i.e. smaller differences in estimates between the 2 observers) than the RPQ estimates (paired $t$-test, $\mathrm{p}=0.046$ ). Most of the difference between methods was seen in estimates of the less common species ( $<10 \%$ cover); Table 1 shows that the RPQ method was significantly less repeatable for the rare species but not the abundant ones. Mean differences between values were small, but the ranges were rather large, especially for the RPQ method (Table 1).

We also analyzed whether either estimation method showed a consistent bias in giving high or low abundance values relative to the other. Fig. 2 shows that while there is a strong correlation between the mean values for the 2 methods, the mean of the 2 visual estimate values was usually lower than the mean of the $2 \mathrm{RPQ}$ values; this was the case in $52 / 85$ speciesoccurrences, or $52 / 69$ if the species missed by RPQ are eliminated. The laboratory data, below, shed light on this difference.

Table 1. Repeatability of visual and RPQ estimates in the field. Values are the mean and range of differences in percent cover between estimates for the 2 observers using each method, summed over all species-occurrences. Rare species (<10\% cover) included 45 species-occurrences, abundant species ( $>10 \%$ ) included 24 . Numbers of species-occurrences whose estimates were more repeatable using each of the 2 methods are also given. Some ties occurred where both methods were equally repeatable. Statistical comparisons of mean differences used paired $t$-tests, comparisons of the number of more repeatable occurrences used a sign test

\begin{tabular}{lccccccc|}
\hline & \multicolumn{3}{c}{ Rare species } & & \multicolumn{3}{c|}{ Abundant species } \\
& $\mathrm{RPQ}$ & Visual & $\mathrm{p}$ value & $\mathrm{RPQ}$ & Visual & $\mathrm{p}$ value \\
\hline Mean (SD) difference & $3.0(2.8)$ & $1.1(1.0)$ & 0.0001 & $7.2(7.0)$ & $6.7(5.1)$ & 0.77 \\
Range of differences & $0-12$ & $0-4$ & - & $0-24$ & $0-16$ & - \\
N more repeatable & 9 & 31 & 0.0005 & 12 & 11 & 0.50 \\
\hline
\end{tabular}




\section{FIELD SAMPLING: RPQ VS. OBSERVERS}

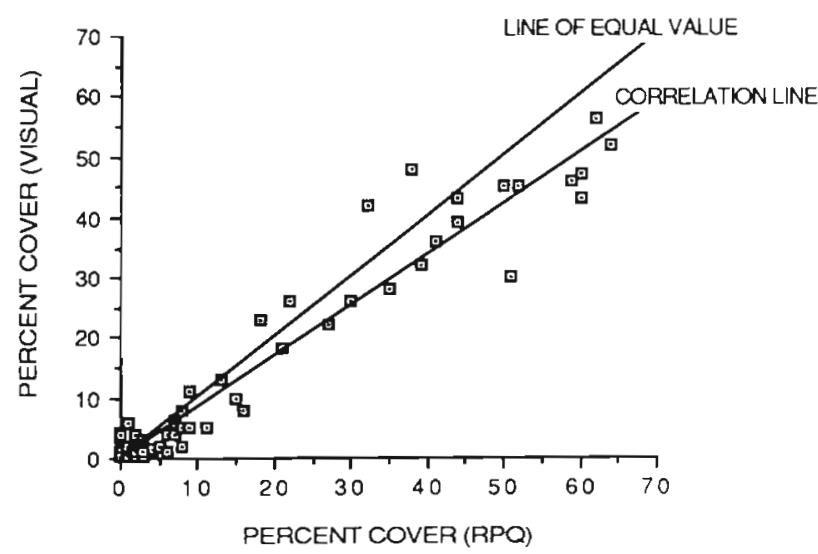

Fig 2. Comparison of values for 85 species-occurrences in the field using RPQ and visual sampling. Each axis represents the mean of the 2 values produced by the 2 observers. Correlation line has a slope of $0.84, r^{2}=0.94$

Times required for sampling a quadrat differed greatly with complexity of the assemblage, but were approximately $10 \mathrm{~min}$ with the visual method and 20 min with the RPQ method.

\section{Simulation}

Table 2 illustrates the accuracy of the sampling methods, i.e. the divergence of the estimates from the digitized values, averaged over all speciesoccurrences. The 3 protocols tested, using the 3 (rearranged) versions of each quadrat, were: (1) the RPQ method using 50 points; (2) the RPQ method using 100 points; and (3) visual estimation by Observer 1 (M.N.D.). These data demonstrate that RPQ estimates (especially using only 50 points) are significantly less accurate than are visual estimates (Kruskal-Wallis 1-way ANOVA and non-parametric multiple compar-

Table 2. Differences among sampling protocols in accuracy, i.e. divergences from the true (digitized) values for each species occurrence in the simulation study (all data pooled, $N=342$ ). Means are calculated from the absolute values of the difference between the digitized value for a given species-occurrence and the estimated value. Kruskal-Wallis 1 -way ANOVA, $p=0.0001$. Letters in the last column are different for significantly different means $(p<0.05$, nonparametric SNK test)

\begin{tabular}{|lrc|}
\hline Comparison & Mean divergence (SD) & SNK \\
\hline Digitized - 50-pt RPQ & $2.3 \%(2.6)$ & A \\
Digitized - 100-pt RPQ & $1.6 \%(1.9)$ & A \\
Digitized - Observer 1 & $0.56 \%(0.54)$ & B \\
\hline
\end{tabular}

Table 3. Differences among observers in sampling accuracy, i.e. divergences from the true (digitized) values in the simulation study. $N=114$ species-occurrences (Replicate A only). Kruskal-Wallis 1 -way ANOVA, $\mathrm{p}=0.23$. Observer 1 was most experienced in visual estimation

\begin{tabular}{|cr|}
\hline Observer & Mean divergence (SD) \\
\hline 1 & $0.56 \%(0.58)$ \\
2 & $0.58 \%(0.57)$ \\
3 & $0.76 \%(0.85)$ \\
\hline
\end{tabular}
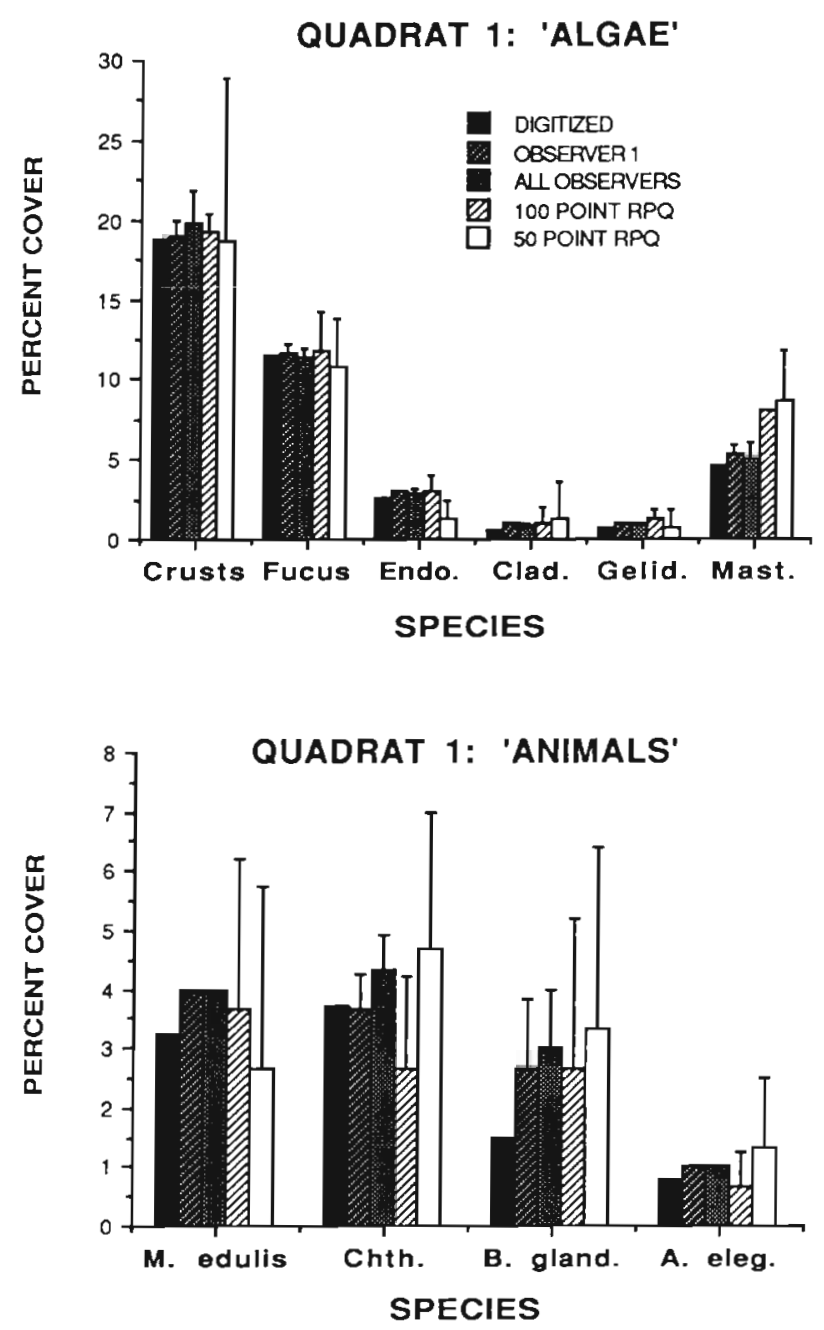

Fig. 3. Comparison of values and variances estimated by each technique for the percent cover of all 'species' in a representative quadrat (one of 30 ) in the simulation. Bars represent either the true (digitized) value, or the mean and 1 standard deviation for the 3 estimates of each speciesoccurrence. Lack of an error bar indicates complete agreement among the 3 estimates for that value (except for the digitized value, which was only measured once). 'Species' in this quadrat were drawn to resemble encrusting algae (Crusts), the algae Fucus, Endocladia, Cladophora, Gelidium, and Mastocarpus, the mussel Mytilus, the barnacles Chthamalus and Balanus, and the anemone Anthopleura 
isons). The accuracy of the $2 \mathrm{RPQ}$ protocols did not differ significantly from each other. Maximum divergences from digitized values followed the same trend in accuracy; visual estimates diverged by up to $5 \%$, RPQ with 100 points by up to $16 \%$, and with 50 points by up to $22 \%$.

Table 3 illustrates differences in sampling accuracy among the 3 observers (using only the 1 version of each quadrat used by all 3 observers). The most experienced observer showed the smallest degree of divergence from the true value, but these differences were not significant.

Fig. 3 presents the results from 1 representative simulated quadrat to illustrate that the sampling protocols also differed widely in repeatability, i.e. variance among the estimates from the 3 replicate versions of each quadrat (except for the 'All Observers' bar, which represents the mean and variance among the 3 observers using Version $A$ of each quadrat only). For all the species-occurrences, the 3 protocols differed significantly in variance (Table 4 ) and multiple comparisons indicated that each protocol is significantly different from the others. The variance among the 3 observers was not included in this analysis because of non-independence from the Observer 1 data, but it was substantially lower $(0.6 \pm 0.7)$ than either $R P Q$ variance. Thus again, RPQ (50 points) was the most variable, and visual estimation by Observer 1 was the least.

Other analyses tested how well the different protocols estimated the abundance of common vs rare species (Table 5). Species that covered less than $2 \%$ (digitized values) of the quadrat (156 out of 342 species-occurrences) were poorly estimated by both RPQ and visual estimation (Table 5A). The RPQ method missed many of the rare species-occurrences (60\% for 50-point RPQ, $45 \%$ for 100-point). When a
Table 4. Differences among sampling protocols in repeatability, i.e. variances among the 3 replicate versions of each quadrat. Mean standard deviations are calculated from all the standard deviations among the 3 replicates for each speciesoccurence $(\mathrm{N}=114)$. Kruskal-Wallis test, $\mathrm{p}=0.0001$. Letters in the last column are different for significantly different means $(p<0.05$, non-parametric SNK test $)$

\begin{tabular}{|lcc|}
\hline Method & Mean standard dev. (SD) & SNK \\
\hline 50 -pt RPQ & $2.6(2.2)$ & A \\
100-pt RPQ & $1.6(1.4)$ & B \\
Observer 1 & $0.3(0.4)$ & $\mathrm{C}$ \\
\hline
\end{tabular}

point did contact a rare species it usually resulted in an overestimation, since the lowest percent cover that could be produced by RPQ was $2 \%$ (50 points) or $1 \%$ (100 points). Visual estimation was more reliable in terms of 'finding' rare species in each quadrat ( 0 missed), but most percent cover values were overestimated. The visual protocols overestimated coverage more of the time but to a lesser degree than the RPQ protocols (Table 5A).

Coverages of abundant species (186 out of 342 species-occurrences) were proportionately better estimated by both visual estimation and RPQ techniques (Table 5B). The RPQ method continued to miss species with covers under $5 \%$ (19 of the 20 'missed' occurrences were of species $<5 \%$ ). Again, all methods tended to overestimate the true value more often than expected (Table 5B, Fig. 3). The 4 protocols differed in their frequency of overestimation (Chi-Square, p < 0.02); 50-point RPQ overestimated the least often but to the greatest degree, and Observer 1 overestimated the most often but to the smallest degree (Table 5B). The total amount of overestimation (frequency times

Table 5. Comparison of accuracy of sampling and direction of bias for rare vs common species, using each of 4 estimation protocols on the simulated quadrats. $N=$ number of species occurrences per category. Each mean = mean deviation of the estimate from the digitized value, either for total sample, for overestimated values, or for underestimated values. The latter does not include species-occurrences that were missed altogether in the RPQ method (i.e. no point contacted that species). Digitized values were almost always non-integers, making it unlikely that observer values would be in precise concurrence

\begin{tabular}{|c|c|c|c|c|c|c|c|}
\hline \multirow{2}{*}{ Comparison } & \multicolumn{2}{|c|}{ Total sample } & \multicolumn{2}{|c|}{ Overestimations } & \multicolumn{2}{|c|}{ Underestimations } & \multirow{2}{*}{$\begin{array}{l}\text { Numbe } \\
\text { missed }\end{array}$} \\
\hline & $N$ & Mean (SD) & $\mathrm{N}$ & Mean (SD) & $N$ & Mean (SD) & \\
\hline \multicolumn{8}{|c|}{ A. Rare species ( $<2 \%$ cover digitized) } \\
\hline Digitized - 50-pt RPQ & 156 & $1.2(1.1)$ & 63 & $1.9 \quad(1.4)$ & 0 & - & 93 \\
\hline Digitized - 100-pt RPQ & 156 & $0.80(0.78)$ & 72 & $1.1(1.0)$ & 14 & $0.28(0.23)$ & 70 \\
\hline Digitized - All observers & 156 & $0.36(0.35)$ & 145 & $0.37(0.35)$ & 10 & $0.14(0.07)$ & 0 \\
\hline Digitized - Observer 1 & 156 & $0.33(0.29)$ & 148 & $0.34(0.29)$ & 7 & $0.09(0.05)$ & 0 \\
\hline \multicolumn{8}{|c|}{ B. Common species ( $>2 \%$ cover digitized) } \\
\hline Digitized - 50-pt RPQ & 186 & $3.3(3.1)$ & 104 & $4.0 \quad(3.6)$ & 62 & $2.3(2.3)$ & 20 \\
\hline Digitized - 100-pt RPQ & 186 & $2.3(2.3)$ & 116 & $2.6(2.6)$ & 67 & $1.6 \quad(1.6)$ & 3 \\
\hline Digitized - All observers & 186 & $0.86(0.80)$ & 118 & $0.96(0.83)$ & 68 & $0.69(0.72)$ & 0 \\
\hline Digitized - Observer 1 & 186 & $0.76(0.62)$ & 134 & $0.91(0.62)$ & 52 & $0.37(0.41)$ & 0 \\
\hline
\end{tabular}


degree) was 2 to $3 \times$ greater for the RPQ method than for the visual estimates. These data suggest that in the field sampling (Fig. 2), the visual estimates may have been closer to the true value than were the RPQ estimates.

The average times required to census quadrats differed less in the simulation than in the field. Visual estimation and 50-point RPQ took comparable amounts of time ( 3 to $4 \mathrm{~min}$ each), while 100 -point RPQ took longer ( 5 to $6 \mathrm{~min}$ ).

\section{DISCUSSION}

Our results suggest that visual estimation is a legitimate technique for estimating percent cover, producing data that are accurate and repeatable (not only by the same observer, but also among different observers). To our surprise, the RPQ method, although theoretically objective, was significantly less accurate than visual estimation. Single RPQ counts often gave numbers highly different from the true value, although as is true for any probabilistic sampling scheme, repeated counts converged on the truth. Since quadrats are usually sampled only once per sampling period in the field, and since accuracy not just for the population but for each quadrat may be important (e.g. to look for changes through time in permanent quadrats), the accuracy and reliability of a single census time are crucial in assessing the relative strengths and weaknesses of the 2 methods. By this criterion, the visual estimation method is better than the RPQ method.

Using 50 to 100 points in RPQ sampling has been adopted as a standard without adequate testing. We found that increasing the number of random points from 50 to 100 improved the accuracy and decreased the variability of RPQ estimates. However, sample size calculations show that prohibitively large numbers of points are needed to distinguish even moderate differences in percent cover values at a $95 \%$ confidence level. For example, for a species with $40 \%$ cover, only 92 points are needed to distinguish 40 from $50 \%$, but 369 points are needed to distinguish 40 from $45 \%$; for a species with $3 \%$ cover, 1118 points are needed to distinguish 3 from $4 \%$. The number of points needed to ensure contact with rare species is extremely high. Rare species ( $<5 \%$ cover) were often missed by the RPQ method, both in the field and simulation, whereas the visual method was more sensitive, i.e. consistently effective in noting all species present in a quadrat. Meese \& Tomich (1992) found that the threshold of detectability for their point-intercept method was $\sim 1 \%$. Researchers who use point-intercept methods in the field usually list 'rare' species (those not contacted by points), adding to sampling time.
Visual estimation and 50-point RPQs required comparable amounts of time when using 2-dimensional, simulated quadrats. In the field, however, the RPQ method took roughly twice as long. When organisms were multi-layered (as is often the case), for every random point the frame had to be picked up and the upper layer of plants moved aside to see the understory [more sophisticated frames such as that of Foster et al. (1991) alleviate this problem]. Photographic estimation techniques encounter similar problems in such systems. With visual estimates, each layer of organisms can be estimated without moving the quadrat, and then the upper species are held out of the way to sample lower layers. Doubling the number of points to increase RPQ accuracy in the simulation required a substantial increase in sampling time. Since time is always a factor in field sampling, and especially in intertidal research, the improvements gained by increased point number must be weighed against the reduction in number of quadrats that can be sampled with time. In addition, using 100 points still produced RPQ estimates that were significantly less accurate, more variable, and more biased towards overestimation than the visual estimates (Tables 2, 4 \& 5).

The visual method is in some ways more difficult to perform, since the sampler needs to concentrate on the distributions of various species rather than simply identifying and recording the species under each point. The usefulness of the visual method is probably limited to relatively small quadrat sizes $\left(\leq 1 \mathrm{~m}^{2}\right)$, since areas larger than this are difficult to 'integrate' mentally (written tallies of grid squares filled could facilitate this integrative process). In addition, while results using different grid sizes have not been tested explicitly, our experience indicates that it is important to use a relatively large number of small subdivisions to aid the eye in censusing scattered or low-coverage species. Use of large subdivisions does not give the eye an accurate enough reference frame. In addition, a visual method should not rely on creation of any complex decision rules (see 'Methods'); these are likely to reduce sampling efficiency and increase amongobserver variability. Pre-fieldwork training on the sort of computer images used in this study (Fig. 1) may help researchers become aware of problems and potential biases in this technique. Meese \& Tomich (1992) found their visual technique to be more variable between observers than either point-intercepts or digitizing, but they did not describe the use of quadrat subdivisions, which we consider critical. For the visual technique to be justifiable, it must be used carefully. However, our results show that even relatively inexperienced observers (Obs, 2 and 3, Table 3) can produce visual estimates that are not significantly different in their accuracy from estimates performed by an experienced 
observer (Obs, 1), even though 'training' was minimal. Not surprisingly, variability among different observers was greater than within-observer variance, but both variances were still lower than with the RPQ method.

A major argument against the use of the visual estimation technique is that it is subjective and thus has the potential to be biased. This is especially true if the observer 'wants' a given outcome (e.g. predicted from a hypothesis), but we suggest that this could be a problem with any estimation technique. For example, we found that bias (notably the tendency to overestimate abundance of species with low percent covers) is similar across different observers using visual estimates, but to our surprise exists for the RPQ method as well; this may stem from an unconscious desire for a point that is only close to a species to be recorded as a 'hit'. Greig-Smith (1964) and Bonham (1989) concur that point-intercept methods have a subjective component, in deciding whether a pin of finite size (as opposed to a theoretical 'point') actually touches a species; points or pins tend to overestimate percent cover (Goodall 1952). A researcher using the $R P Q$ method may not count an abundant species touched by the edge of a point, but include a rare species that is barely touched. Observers using visual estimates may be more confident that they are including all species and thus be less likely to consciously or unconsciously compensate in their estimates. Other errors are introduced if pins are not lowered (or points are not viewed) at a constant angle. Meese \& Tomich (1992) found that evenly spaced dots were more subject to observer bias than were random ones. Thus while bias must constantly be guarded against, the visual method is not alone in this need. Both methods require training to reduce subjectivity and the role of known biases; the need for training is not a reason to exclude a technique.

Concern about subjectivity or different biases among research teams might cause some investigators to choose a point-intercept method when the appearance of bias is unacceptable, e.g. in studies of oil spill damage. If different teams must perform pre-spill and postspill sampling, are visual estimates valid? We would argue that they are, since we found that (1) amongobserver variation in visual estimates was low (and accuracy of estimates fairly high), even with minimal training; and (2) errors inherent in RPQ sampling could be as bad (or worse) among teams as were the large errors noted during $\mathrm{RPQ}$ sampling within our team. With either method, post-census cross-calibrations could be performed, e.g. by a cooperative field or simulation study with the 2 teams, where some quadrats are sampled by each team and estimates compared (as in this study). If 2 or more teams are used during a given sampling period, an experimental design incorporating repeated sampling and interpenetrating sub- samples (Cochran 1977, p. 388) could be used to quantify interteam differences.

The primary advantages of the widely used RPQ method are its (relative) objectivity and the fact that its accuracy can be theoretically estimated, making it appealing for the purposes of statistical analysis. Such probabilistic sampling methods assume that there is sampling error, but allow conclusions to be drawn even though sampling is not complete; precision can be improved by increasing the number of sampling points or the number of quadrats. In contrast, the visual estimation method involves 'measuring' everything within a quadrat; it is not a probabilistic method (except in having replicate quadrats to sample the whole population in an area). The same is true for digitizing from photographs. Since what is ultimately important in any method is accuracy, if confidence in the visual estimates can be high, then there is no need for probabilistic sampling within a quadrat. Our study has shown that the measurement error using the visual technique is less than the error inherent in sampling using the point-intercept method.

The photographing and digitizing method recommended by Meese \& Tomich (1992) as being the most repeatable between and among observers is a clearly valid alternative, in some systems, to the visual technique recommended here. For 2-dimensional, monolayered organisms which can readily be distinguished in photographs, this method is rapid (in the field, where time is most limited) and precise (although expensive, and subject to parallax and camera error). However, for multilayered systems or where there are many species of similar morphology, the visual technique has clear advantages. We concur with Meese \& Tomich that the best compromise may be to train observers in using careful visual techniques, and to supplement these observations with photographs.

In conclusion, in this study the inaccuracy, variability, and degree of bias of visual estimates were much lower than for RPQ estimates, demonstrating that (trained) observer subjectivity contributes relatively little to error in estimating percent cover of organisms compared to the errors inherent in RPQ sampling. In general, within-quadrat sampling variance, by whatever method, is likely to be small relative to amongquadrat variance in naturally heterogeneous assemblages. One can therefore argue that, at least in patchy communities, increasing sample size by using the more rapid (and more accurate) visual technique is highly desirable for reducing overall error; i.e. extensive sampling is more important than intensive (also noted by Meese \& Tomich 1992). In the end, the visual technique will give a much more accurate representation of relative percent covers of organisms than will using the more 'objective' RPQ technique. 
Acknowledgements. This study was funded by the Minerals Management Service. Space at the Friday Harbor Laboratories was provided by Dr A. O. D. Willows, and access to field sites by the National Park Service. We thank D. Pentcheff for computer assistance, A. Sewell for doing a set of estimates, J. Skalski for sample size formulas and conversations about. sampling, and D. Duggins, E. Grosholz, and several anonymous reviewers for commenting on the manuscript.

\section{LITERATURE CITED}

Bonham, C. D. (1989). Measurements for terrestrial vegetation. John Wiley and Sons, New York

Cochran, W. G. (1977). Sampling techniques, 3rd edn. John Wiley and Sons, New York

Dethier, M. N. (1984). Disturbance and recovery in intertidal pools: maintenance of mosaic patterns. Ecol. Monogr. 54:

This article was presented by M. S. Foster, Moss Landing, California, USA
$99-118$

Foster, M. S., Harrold, C., Hardin, D. D. (1991). Point vs. photo quadrat estimates of the cover of sessile marine organisms. J. exp. mar. Biol. Ecol. 146: 193-203

Goodall, D. W. (1952). Some considerations in the use of point quadrats for the analysis of vegetation. Aust. J. Sci. Res. 5 $1-41$

Greig-Smith, P. (1964). Quantitative plant ecology, 2nd edn Butterworths, London

Jones, W. E., Bennell, S., Beveridge, C., McConnell, B., MackSmith, S., Mitchell, J. (1980). Methods of data collection and processing in rocky intertidal monitoring. In: Price, $\mathrm{J}$. H., Irvine, D. E. G., Farnham, W. F. (eds.) The shore environment. Vol. 1: Methods. Academic Press, London, p. $137-170$

Meese, R. J., Tomich, P. A. (1992). Dots on the rocks: a comparison of percent cover estimation methods. J. exp. mar. Biol. Ecol. 146: 193-203

Manuscript first received: January 8, 1993 Revised version accepted: March 26, 1993 